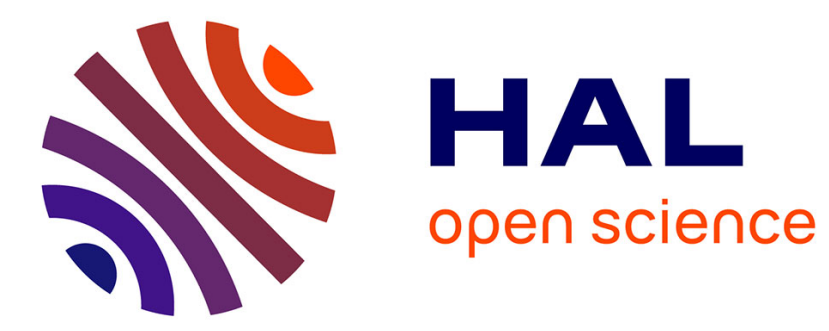

\title{
Le lignage aristocratique en Provence au XIe siècle $\left(14^{\circ}-15^{\circ}\right.$ siècles $)$ \\ Martin Aurell
}

\section{To cite this version:}

Martin Aurell. Le lignage aristocratique en Provence au XIe siècle $\left(14^{\circ}-15^{\circ}\right.$ siècles $)$. Annales du Midi : revue archéologique, historique et philologique de la France méridionale, 1986, 98 (174), pp.149-163. 10.3406/anami.1986.5708 . halshs-01306173

\section{HAL Id: halshs-01306173 https://shs.hal.science/halshs-01306173}

Submitted on 22 Apr 2016

HAL is a multi-disciplinary open access archive for the deposit and dissemination of scientific research documents, whether they are published or not. The documents may come from teaching and research institutions in France or abroad, or from public or private research centers.
L'archive ouverte pluridisciplinaire HAL, est destinée au dépôt et à la diffusion de documents scientifiques de niveau recherche, publiés ou non, émanant des établissements d'enseignement et de recherche français ou étrangers, des laboratoires publics ou privés. 


\section{Le lignage aristocratique en Provence au Xle siècle}

Martí Aurell i Cardona

\section{Citer ce document / Cite this document :}

Aurell i Cardona Martí. Le lignage aristocratique en Provence au Xle siècle. In: Annales du Midi : revue archéologique, historique et philologique de la France méridionale, Tome 98, №174, 1986. Le lignage aristocratique en Provence... pp. 149163;

doi : 10.3406/anami.1986.5708

http://www.persee.fr/doc/anami_0003-4398_1986_num_98_174_5708

Document généré le 14/03/2016 


\title{
Résumé
}

Corollaire de l'effondrement du pouvoir central, des structures lignagères se mettent en place au sein de l'aristocratie provençale dès les années 1020-1030. Une étude quantitative du cartulaire de SaintVictor montre l'importance de ce phénomène : au Xle siècle, plus de la moitié de ses actes ont été passés par des familles élargies. Les comportements familiaux qui découlent du lignage sont les suivants : natalité pléthorique, nuptialité élevée, naissance de sous-lignages et primogéniture. Un rôle nouveau est dévolu à la parenté dans les mentalités religieuses.

\begin{abstract}
As as corollary of the break-down of central power, lineage structures fell into place within the Provençal aristocracy by the years 1020-1030. A quantitative study of the Saint-Victor cartulary shows the importance of this phenomenon : during the Xlth century more than half of its acts were drawn up by extended families. The kinds of family behavior that proceeded from lineage were the following : a very high birthrate, a high rate of marriages, the emergence of sub-lineages and of primogeniture. A new role was vested in kinship in religious mentalities.
\end{abstract}

\section{Zusammenfassung}

Als Begleiterscheinung des Zusammembruchs der Zentralmacht entstehen seit den Jahren 1020-1030 im provenzalischen Adel Geschlechterstrukturen. Eine quantitative Untersuchung des Cartulariums von Sankt Victor fördert die Bedeutung dieser Erscheinung zutage : im 11. Jh. sind mehr als die Hälfte der Rechtshandlungen von erweiterten Familien gezeichnet worden. Folgende Verhaltensweisen der Familien ergeben sich aus ihrer Selbstauffassung als Geschlecht : Geburtenfülle, hohe Heiratsraten, Entstehen von Seitengeschlechtern und Primogenitur. In den religiösen Denkweisen kommt der Elternschaft eine neue Rolle zu. 


\section{LE LIGNAGE ARISTOCRATIQUE EN PROVENCE AU XI' SIĖCLE}

Que l'étude de la parenté ait renouvelé la problématique des historiens du Moyen Age au cours des dernières décennies n'a pas besoin de démonstration ${ }^{1}$. Les recherches effectuées dans ce domaine sont, en grande partie, tributaires de l'interdisciplinarité. L'apport de l'école juridique d'entre les deux guerres facilita à $\mathbf{M}$. Bloch la rédaction des passages précurseurs, consacrés aux "liens du sang ", dans La Société féodale parue en 19392. L'influence de l'ethnologie a considérablement marqué, au cours des années 60 et 70 , les monographies des médiévistes français ${ }^{3}$. Dans un premier temps, ceux-ci ont tenté de saisir la famille comme une réalité sociale. Ils ont, notamment, décrit les mécanismes qui conditionnent sa nucléarisation ou son élargissement ${ }^{4}$. Mais, de plus en plus, ils s'intéressent à la place que cette institution occupe dans l'imaginaire de l'homme médiéval5.

L'acquis méthodologique de ces travaux enrichit l'interprétation des sources provençales du XIe siècle. Autour de 1020, un certain individualisme des nobles à l'égard de leurs familles, qui transparaît à la lecture des chartes du $\mathrm{X}^{\mathrm{e}}$ sièclé, est remis en question. Au lendemain de l'an mil, la Provence s'enlise dans une période de décomposition de la puissance comtale. Les sires brisent l'ordre social que la dynastie des descendants de Boson avait instauré entre Rhône et Durance. Ils se livrent à des guerres privées dont le corollaire est l'effondrement des institutions centrales. Le prince qu'ils combattent n'est plus à même de fournir sa protection aux membres de

* 14, avenue Jules-Isaac, 13100 Aix-en-Provence.

1. Il serait superflu de citer ici les nombreux ouvrages et articles relatifs à la famille médiévale publiés dernièrement. Le lecteur trouvera ces références dans l'orientation bibliographique proposée par R. Fossier, Enfance de l'Europe. Aspects économiques et sociaux, Paris, 1982, p. 17-19.

2. Pages 183-208 de la réédition de 1978 .

3. A. Guerreau-Jalabert, Sur les structures de parenté dans l'Europe médiévale, dans Annales. Economies, Sociétés, Civilisations, 1981, p. 1028-1049, où cette collaboration avec les anthropologues est défendue avec un acharnement un tant soit peu excessif.

4. G. Duby, Hommes et structures du Moyen Age, Paris-La Haye, 1973, p. 420-421.

5. M. Sot, Historiographie épiscopale et modèle familial en Occident au IXe siècle, dans Annales. Economies, Sociétés, Civilisations, 1978, p. 433-449 ; B. Guenee, Les généalogies entre l'histoire et la politique : la fierté d'être capétien, en France, au Moyen Age, ibidem, p. 450-477.

6. A cette époque, les grandes familles partagent des patrimoines entre les enfants (P.-A. AMARGIER, Aux origines de la famille des vicomtes de Marseille, dans Le Moyen Age, 1964, p. 161-178). Chacun est libre de se dessaisir d'une partie de son domaine sans le consentement de ses parents. 
l'aristocratie. Ce sont alors les solidarités familiales qui prennent le relais. Chaque maison doit désormais tirer ses ressources d'un patrimoine propre qu'il importe de sauvegarder en indivision.

Cette mutation est d'autant plus facile à cerner que le XIe siècle provençal se caractérise par une augmentation considérable du nombre des actes de la pratique. Entre les années 1030 et 1070, le flot de donations vers le monastère de Saint-Victor, dont le cartulaire demeure notre source principale, atteint son apogée. Une abondante documentation foisonne de renseignements sur les nouvelles formes que la famille nobiliaire adopte au XI ${ }^{e}$ siècle. Les actes de la période permettent, ainsi, d'approcher ces transformations et de cerner les milieux où elles interviennent. Ils constituent, d'ailleurs, une source de premier ordre pour décrire les mécanismes qui régissent la vie du lignage. Dressés dans les scriptoria monastiques, ils témoignent de l'influence de ce système de parenté dans les mentalités religieuses.

\section{Genèse et milieux d'accueil du lignage}

R. Fossier utilisa, pour la première fois en 1968, une méthode qui permit de comprendre, avec la précision de l'histoire quantitative, l'ampleur du resserrement de la famille dans le lignage 7 . Il décompta le nombre des actes passés par des personnes individuelles, par des couples ou par l'ensemble de la famille élargie dans la Picardie du $\mathrm{X}^{e}$ et du $\mathrm{XI}^{e}$ siècles. Les données que l'application de cette méthode statistique permet de tirer du fonds victorin ont été groupées dans le tableau 1. Une interprétation diachronique de ses chiffres traduit le triomphe du lignage dans la décennie 1020-1030, où il prend le dessus sur toutes les autres formes de parenté. Cette période correspond au début des guerres qui se déroulent en Provence pour récupérer Fos et à la déstabilisation de l'ordre public qui s'ensuit. Tout au long du XIe siècle, la famille élargie ( $41 \%$ des cas étudiés) est reine. Son importance ne fait que s'accroître si nous l'additionnons aux frérèches $(9,5 \%)$. En contrepartie, les individus agissant seuls (14\%) et les couples avec ou sans leurs enfants $(35,5 \%)$. représentent $49,5 \%$ des exemples que fournit le cartulaire de Saint-Victor ${ }^{8}$. Ces chiffres, dans lesquels le lignage l'emporte pour plus de la moitié, semblent contredire, de prime abord, les conclusions qui ont été proposées pour la Picardie, pour la Catalogne et pour le Bas-Languedoc. Ainsi, R. Fossier constate la défaite de la famille large dans la région qui a fait l'objet de son étude. Ses recherches lui ont permis d'évaluer à $83 \%$ le total des actes conjugaux ou individuels passés au

7. La terre et les hommes en Picardie jusqu'à la fin du XII'e siècle, Paris, 1968, p. 264-265.

8. Le chartrier de Saint-Victor fournit des données semblables. Sur 49 contrats, 4 ont été passés par des individus seuls, 6 par des couples, 14 par des familles étroites, 5 par des frères et 20 par des familles élargies : P. AMArgler éd., Chartes inédites du fonds de Saint-Victor de Marseille, thèse de $3^{e}$ cycle multigraphiée, Aix, 1967. 


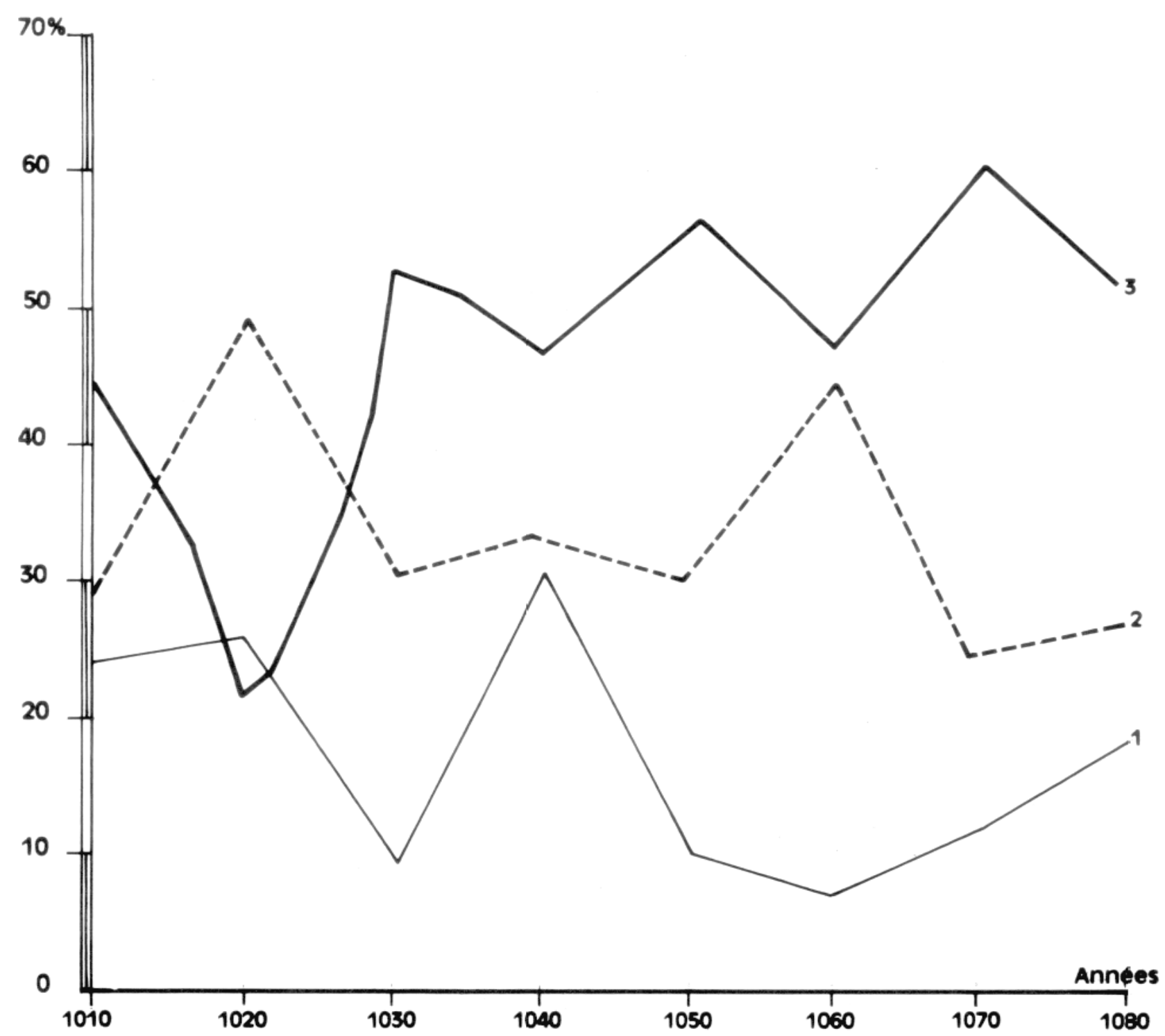

1 : Individus (hommes ou femmes seuls). 2 : Famille étroite et couples. 3 : Famille élargie et frérèches.

\begin{tabular}{|l|r|r|r|r|r|r|r|r|r|}
\cline { 2 - 9 } \multicolumn{1}{c|}{} & $\begin{array}{r}1000- \\
1009\end{array}$ & $1010-$ & $1020-$ & $1030-$ & $1040-$ & $1050-$ & $1060-$ & $1070-$ & Totaux \\
\hline Homme seul & 4 & 4 & 4 & 12 & 4 & 5 & 5 & 5 & 43 \\
Femme seule & 1 & 3 & & 11 & 1 & 2 & 3 & 2 & 13 \\
Couple & 3 & 3 & 6 & 6 & 6 & 12 & 5 & 5 & 46 \\
Famille étroite & 3 & 10 & 6 & 19 & 9 & 33 & 10 & 5 & 95 \\
Frères & 2 & & 2 & 5 & 10 & 8 & 7 & 4 & 38 \\
Famille élargie & 7 & 6 & 19 & 30 & 17 & 39 & 30 & 15 & 163 \\
\hline Totaux & 20 & 26 & 37 & 73 & 47 & 99 & 60 & 36 & 398 \\
\hline
\end{tabular}

Tableau 1. - Les donateurs des actes de Saint-Victor. Source: CSV, t. 1. 
$\mathrm{X}^{\mathrm{e}}$ siècle, à $64 \%$ pendant la première moitié du XIe et à $79 \%$ durant la seconde 9 . Les pourcentages de P. Bonnassie sont assez proches : la famille nucléaire réalise $88 \%$ des aliénations de biens au $\mathrm{X}^{\mathbf{e}}$ siècle. En outre, les testaments catalans de l'époque ne contiennent guère de legs à des collatéraux. Pour le XIe siècle, cet historien a pu établir l'apparition des liens lignagers sans faire appel à une pesée quantitative10. De son côté, M. Bourin observe la rareté des " complexes familiaux " dans l'aristocratie biterroise du $\mathrm{XI}^{\mathrm{e}}$ siècle ; les consanguins autres que le conjoint et les enfants interviennent uniquement dans 10 à $16 \%$ des donations pieuses ${ }^{11}$. En revanche, nos sources ne nous permettent pas d'affirmer d'emblée la victoire de la cellule conjugale dans le contour de la Méditerranée, telle qu'elle a été proclamée à maintes reprises ${ }^{i 2}$. Quelques remarques critiques sur la nature de nos textes permettront de comprendre cette force apparente des structures lignagères dans la Provence du XIe siècle.

Les données tirées du fonds victorin proviennent, en fait, d'une quantité considérable de donations qui émanent de la très grande aristocratie provençale. Les Reillanne, les Marignane et, surtout, les vicomtes de Marseille sont majoritairement représentés dans le cartulaire de Saint-Victor. Or, la recherche historique des dernières décennies a bien établi que le resserrement de la famille s'est principalement pratiqué dans la haute noblesse ${ }^{13}$. Même la fonction comtale semble assumée en frérèche au cours du XI $\mathrm{XI}^{\mathbf{e}}$ siècle. Bertran 1er (1019-1051) et Jaufre 1er (1015-1062) gouvernent la Provence ensemble. Guilhem VI (1053-1062) et Jaufre II (1053), enfants de Bertran, agissent de même. La logique du système veut que la contraction lignagère soit plus précoce et intense au sein de la famille de ceux qui ont reçu la mission de commander et de punir. Ces personnages prennent, à l'époque de la crise politique, les armes pour imposer la loi dans le domaine qui leur est échu. Ce genre de vie militaire comporte des dépenses onéreuses. C'est pourquoi les membres de chaque maison doivent veiller à la conservation intégrale de leur patrimoine foncier. Les guerres privées dans lesquelles ils se

9. Op. cit., p. 264-266 et 271-273.

10. La Catalogne du milieu du Xeà la fin du XIe siècle, croissance et mutations d'une société, Toulouse, 1975, p. 266-269 et 547 .

11. " La règle est et demeure la famille conjugale, avant et après la crise, sur les manses et dans le castrum "; M. Gramain-DerRuau, Villages et communautés villageoises en Bas-Languedoc (vers 950 vers 1350): l'exemple biterrois, thèse d'Etat multigraphiée, Paris I, 1979, p. 204. Nous nous permettons, toutefois, de présenter quelques remarques critiques quant à l'établissement de ses tableaux. Pour M. Bourin, en effet, l'apposition d'un signum dans un acte de donation par les parents lointains n'implique pas la légalisation de leur droit sur le bien cédé (op. cit., p. 208-209). Cependant, nous considérons que l'apparition de plusieurs consanguins dans la liste des souscripteurs d'une transaction ne s'explique que par un certain droit de regard sur le patrimoine. Les sociétés traditionnelles ont une conception de la propriété bien plus souple que la nôtre, forgée dans le positivisme juridique, et le fait de figurer dans la liste des souscripteurs prend très souvent sa raison d'être dans une certaine jouissance que chacun avait sur le bien qui était abandonné par un membre de sa propre famille.

12. Notamment par P. Toubert. Les structures du Latium médiéval, Rome, 1973, p. 711.

13. G. DuBY, op. cit., p. 420 ; J. HeERs, Le clan familial au Moyen Age, Paris, 1974, p. 39-40. 
trouvent impliqués les poussent à serrer les rangs derrière un chef de clan pour mener une action commune. Cette militarisation des couches supérieures explique que nos textes limitent l'apparition du lignage aux groupes les plus favorisés de la société provençale. Ce sont les seuls représentants d'une noblesse rurale qui se trouvent dans les actes du monastère marseillais. Il paraît que les documents catalans, picards et biterrois concernent un échantillon sociologique plus large. La cause du décalage des chiffres qu'ils nous livrent s'explique donc par cette différence de perspective ${ }^{14}$.

Ces impressions sont pleinement confirmées par le décompte des contrats passés par des particuliers avec l'archevêque et le chapitre d'Arles, conservés dans l'Authentique de la cathédrale Saint-Trophime (tableau 2). Ses chiffres diffèrent radicalement des données du fonds de Saint-Victor. Cette foisci, les individus représentent plus du cinquième des contractants pour toute la période 950-1090. Fait plus révélateur, le groupe des conjoints avec leurs enfants est largement majoritaire (les deux tiers). Enfin, la part négligeable des frérèches et de la famille élargie ne compte guère qu'à partir des années 1050. Ces renseignements se rapprochent bien plus de ceux que nous livrent les chartriers et les cartulaires des autres régions méditerranéennes. Le fait que la majorité des contractants proviennent de la ville d'Arles est fort significatif. La cité apporte, en effet, à l'individu une autre protection que celle de l'entourage familial. Cet encadrement de la communauté urbaine se matérialise dans le paysage par les remparts qui la préservent des attaques extérieures ${ }^{15}$. Beaucoup des citadins et des alleutiers qui passent des contrats de complant, qui reçoivent des bénéfices de l'archevêque d'Arles ou qui abandonnent leurs maisons au chapitre cathédral, n'exercent point de fonction militaire. Ils ne font pas partie, somme toute, de la haute aristocratie qui choisit la guerre comme genre de vie et prend ses parents pour compagnons d'armes. Le fossé qui sépare la vieille noblesse, qui délaisse ses pied-à-terre des villes pour les forteresses récemment érigées dans la campagne, et le milieu urbain, se creuse aussi en matière de comportements familiaux.

L'avènement des structures lignagères demeure, sans aucun doute, le changement le plus notable de l'organisation de l'aristocratie provençale. L'effondrement des institutions publiques en est la cause principale. Les grands désertent la suite comtale et s'installent au centre de leurs domaines où ils se sont emparés du pouvoir de commander et de punir. Cet émiettement du

14. Voir à ce sujet les remarques de R. Fossier (Enfance..., p. 709) sur la prétendue prépondérance de la cellule conjugale du Sud, contrairement à ce qui devrait arriver dans le monde septentrional. Point de lignage dans la paysannerie : M. Bourin, R. Durand, Vivre au village au Moyen Age. Les solidarités paysannes du XI' au XIII'e siècle, Paris, p. 41.42 et 55.

15. L'importance des solidarités villageoises voire urbaines a été mise en relief dans la thèse de M. Bourin ; voir ses remarques sur la protection de l'individu dans un habitat groupé, Villages et communautés..., p. 228-229. 


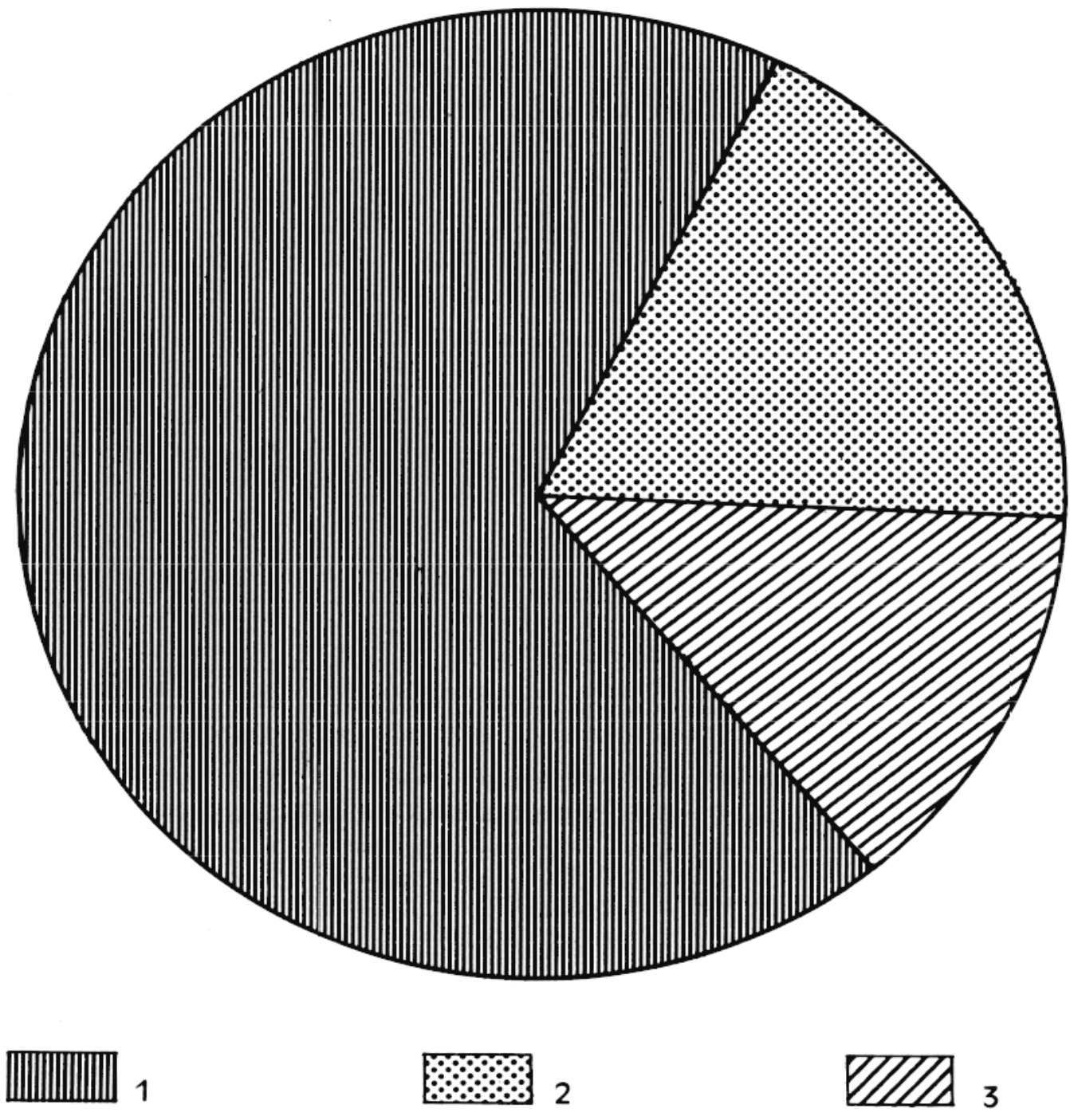

1 : Famille étroite. 2 : Individus. 3 : Familles élargies.

\begin{tabular}{|l|r|r|r|r|}
\cline { 2 - 5 } \multicolumn{1}{c|}{} & $\begin{array}{r}950- \\
1000\end{array}$ & $\begin{array}{r}1000- \\
1050\end{array}$ & $\begin{array}{c}1050- \\
1090\end{array}$ & Totaux \\
\hline Homme ou femme seuls & 2 & 2 & 7 & 11 \\
Couple & 13 & 12 & 6 & 31 \\
Famille étroite & 1 & 4 & 6 & 11 \\
Frères & 1 & 1 & 2 & 4 \\
Famille élargie & & & 4 & 4 \\
\hline Totaux & 17 & 19 & 25 & 61 \\
\hline
\end{tabular}

Tableau 2. - Les parties des contrats du fonds de l'archevêché d'Arles. Source : GCNN, t. 3. 
ban est à l'origine d'une situation trouble où la guerre fait partie du quotidien aristocratique. En milieu rural, la noblesse cherche donc l'encadrement sécurisant de la famille. Il s'ensuit cette cohésion lignagère qui transparaît à la lecture des actes de Saint-Victor. Il n'en va pas de même en ville. Dans le milieu urbain, la force de la famille ne se manifeste que dans le groupe restreint de ces milites civitatis qui choisissent, dans les troubles de la mutation "féodale ", le parti princier. Mais, mis à part cette catégorie de guerriers, qui transmettent de génération en génération le métier des armes à l'intérieur de leur parentèle, la société citadine préserve l'autonomie de l'individu à l'égard de ses consanguins. Les personnes, protégées par les enceintes, connaissent d'autres solidarités sur lesquelles les textes ne nous éclairent guère. Ces liens entre citadins n'apparaîtront au grand jour que dans la deuxième moitié du XII ${ }^{e}$ siècle sous la forme institutionnelle du consulat, réalité juridique qui traduit l'existence antérieure d'une organisation communale. C'est la raison pour laquelle la famille étroite persiste sans solution de continuité dans les cités provençales.

\section{Mécanismes du lignage}

Dans le cadre du lignage, chaque famille essaye de conserver son patrimoine dans son intégralité. Des héritiers trop nombreux pourraient provoquer son morcellement. Il ne semble pas, toutefois, que les conjoints pratiquent la restriction des naissances afin de parer aux partages successoraux. La natalité des maisons aristocratiques apparaît alors sous un jour pléthorique. Le cas de la famille arlésienne de Porcelet, un lignage de guerriers urbains, est très instructif sur ce point ${ }^{16}$. Cinq des enfants de Rostaing Porcelet (1028-1067) nous sont connus; les textes en mentionnent quatre de son fils Guilhem (1056-1094) ainsi que cinq de son petit-fils Jaufre (1101-1149). Les couples de la famille de Châteaurenard comptent, à la même époque, six enfants en moyenne ${ }^{17}$. Or, les documents ne nous montrent que la partie émergée de l'iceberg, eu égard aux pertes considérables dues à la mortalité infantile et au fait que les actes ne nous livrent guère les noms des filles à partir du milieu du XIe siècle. Des familles nombreuses de dix enfants environ apparaissent souvent dans les chartes ${ }^{18}$. Cela confirme l'existence d'un taux de natalité fort élevé en Provence, qui correspond à celui que

16. M. AUReu, Une famille de la noblesse provençale au Moyen Age : les Porcelet, Avignon, Théodore Aubanel, 1986.

17. A. VAnchteIN, La famille des Châteaurenard auX $X I^{e}$ et $X I I^{e}$ siècles, mémoire de maîtrise multigraphié, Aix, 1980, p. 54.

18. Ego Raimbertus, filius Durandi cognomento Blanchi, et uxor mea, nomine Micol, et filii mei Ugo, Pandulfus, Theodatus, Raifambertus, Willelmus, Poncius, Heldebertus, Arbertus, Theofredus, facimus donationem, CSV n' 529 (c. 1040). 
proposent les études entreprises sur la démographie nobiliaire dans d'autres régions 19 .

Plus les membres mâles du lignage sont nombreux, plus sa force militaire augmente. Il paraît même que la politique de limitation de la nuptialité, telle qu'elle est alors systématiquement pratiquée dans le nord de la France ${ }^{20}$, n'est pas la règle générale en Provence. L'exemple de la famille Porcelet est, une fois de plus, riche en renseignements. Trois des quatre enfants de Rostaing Porcelet arrivés à l'âge adulte ont pris femme; seul Bertran, sacriste de la cathédrale, demeure célibataire. De même, deux des trois garçons de Guilhem Porcelet ont eu des descendants. Les vicomtes de Marseille pratiquent une politique matrimoniale identique. Seuls les Baux et les Châteaurenard semblent se comporter comme les nobles septentrionaux ${ }^{21}$. Mais, même pour ces deux maisons, les sources dont on dispose, qui ne permettent pas toujours de reconstituer les filiations aristocratiques de façon précise et mentionnent moins que par le passé les noms de femmes, auraient tendance à cacher une grande partie de ces mariages. Il ne faudrait pas, non plus, voir dans les couvents surpeuplés la proie d'une foule de parents qui cherchent à caser de malheureux cadets sans engager trop de frais. Le milieu monastique n'accueillait pas seulement des oblats en bas âge : au contraire, les monastères recevaient, au XI ${ }^{e}$ siècle, bon nombre de veufs ou de maris qui abandonnaient leurs épouses et leurs enfants pour le cloître22. En ce qui concerne les filles, le modèle est plus clair : on essaye d'en marier le plus possible avec une dot devenue assez mince dès 1050 environ. Ce fait pourrait être corroboré, en Provence, par le nombre très restreint de couvents féminins avant la poussée du monachisme cistercien.

Chaque lignage a donc intérêt à marier tous ses membres ${ }^{23}$. Si les garçons sont nombreux à prendre femme, les effectifs des guerriers provenant de la même famille et s'y trouvant encadrés s'accroissent. La naissance de

19. M. PARISSE, La noblesse lorraine (XI-XIII'e siècle), Lille, 1976, p. 309-310; M. Gramain, Villages et communautés..., p. 205 ; P. BonNASSIE, op. cit., p. 270 , arrive aux mêmes conclusions pour l'ensemble de la société catalane.

20. G. DuBY, Le chevalier, la femme et le prêtre, Paris, 1981, p. 254 et 284.

21. SMYrL, La famille..., p. 10. En ce qui concerne les Châteaurenard, dans la génération de la première moitié du XIe siècle, quatre des sept garçons fondent une famille; dans celle de la seconde moitié du siècle, il n'y a que deux mariés parmi quinze représentants (A. VAINCHTEIN, op. cit., p. 45).

22. Les membres de la famille Porcelet finissent souvent leurs jours parmi les chanoines de la cathédrale d'Arles. Exemples de conversions : CSV $\mathbf{n}^{\circ} 438$ (1027), P. AMARgier, Chartes..., $n^{\circ}$ 51 (1062), L. LABANDE, Chartes de Montmajour aux archives du palais de Monaco, dans Annales de la Société d'études provençales, 1908, $\mathrm{n}^{\circ} 4, \mathrm{H}$. Moris, E. Blanc éd., Cartulaire de l'abbaye de Lérins, Paris, 1883-1905, n 132 (c. 1150). Contra : R. Fossier, Enfance..., p. 338-339.

23. Contrairement à ce qui arrive dans le Latium, où bon nombre d'individus sont voués à " cette éternelle juventus, synonyme d'échec social "(P. Toubert, op. cit., p.767). P. BonNAssie, (op. cit., p. 282) constate un phénomène semblable dans les pays catalans, mais son point de vue ne semble pas partagé par J.-E. Ruz DOMENEC (Systènés de parenté et théorie de l'alliance dans la société catalane (env. 1000-env. 1240), dans Revue historique, 1979, p. 314), d'après lequel les juvenes sont plutôt un modèle culturel qu'une réalité sociale. Cet auteur formule de même la théorie de l'apport des sous-lignages à la puissance militaire du clan. 


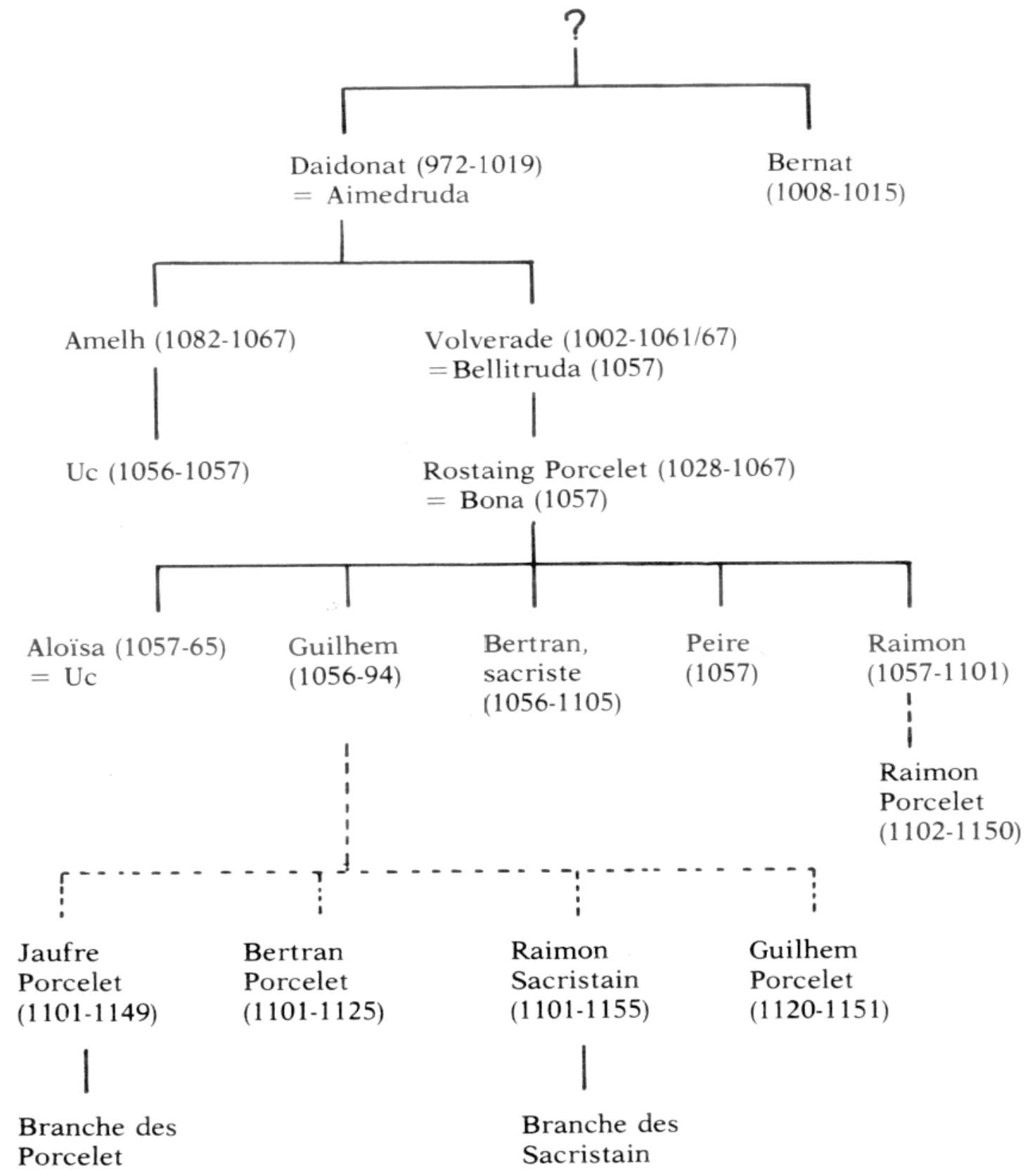

Reconstitution de la filiation des Porcelet au XI' siècle. 
plusieurs sous-lignages qui, moins fortunés que la branche principale de la maison, rentrent dans ses clientèles guerrières, découle de cette prolifération. Quelques personnages dont le cognomen est Daidonat gravitent, à l'époque, autour de la famille Porcelet. La parenté entre les Daidonat - dont le nom est de toute évidence emprunté à celui du fondateur de la maison de Porcelet - et cette dernière famille peut être bien établie : en 1101, Guilhem Daidonat se trouve parmi les neveux de Bertran Porcelet, sacriste. Ce même personnage siège dans le plaid que Volverade, Amelh et Rostaing Porcelet tiennent en Arles au milieu du XIe siècle. Un Daidonat et un Ponç Daidonat figurent également parmi les souscripteurs des actes dressés à la demande des Porcelet. La situation des terres des Daidonat pourrait correspondre au domaine de leurs consanguins : Guilhem Daidonat a, vraisemblablement, ses biens dans la région de Vitrolles, sur les rives de l'étang de Berre, comme il ressort de sa souscription dans une charte par laquelle le seigneur de ce village confirme la cession d'un manse que son fils Gauceran a faite à SaintVictor, avant son départ pour la Terre Sainte. Raimon Daidonat est le dernier représentant de ce sous-lignage et figure, entre 1115 et 1126 , avec Jaufre Porcelet en tant que témoin d'un document du fonds de l'archevêché d'Arles. La disparition subite des Daidonat de la documentation, due probablement au déclassement, corrobore l'hypothèse du rang inférieur de cette lignée. Le fait qu'ils se soient si souvent trouvés dans l'entourage des Porcelet incite, en outre, à penser qu'ils étaient leurs clients. Rien d'étonnant dans cette imbrication entre les fidélités guerrières et les liens de parenté, dont les sources littéraires nous ont livré tant d'exemples 24 .

Le lignage dispose d'un moyen aussi simple que la limitation des naissances ou la restriction des mariages pour s'opposer à la dispersion de sa fortune : la primogéniture25. Chez les Porcelet, l'aîné s'érige en chef militaire à la têt de son clan. Certains privilèges découlent de cette situation. Guilhem Porcelet reçoit, vers 1060, la presque totalité des biens de son père Rostaing au détriment de ses frères et sœurs, et si ses fils Jaufre Porcelet et Raimon Sacristain se partagent au début du XIIe siècle ce domaine, le premier ne s'en taille pas moins la part du lion. Jaufre conserve, en effet, le noyau initial du temporel de sa famille. Ses descendants jouissent par la suite de presque tous les droits de cette maison sur le Vieux-Bourg d'Arles

24. M. Bloch, op. cit., p. 184, et G. DuBy, Les trois ordres..., p. $94-95$ et 202. Le roman occitan de Flamenca présente son héros, Guilhem, frère du comte de Nevers, entouré de deux de ses cousins qui, d'un rang social inférieur au sien, lui servent d'écuyers. D'autres exemples de parentsfidèles dans le Charroi de Nîmes.

25. Le problème du droit d'aînesse dans les successions a été souvent traité dans les termes juridiques du parage. Certains historiens en ont justifié l'apparition par l'unique existence du fief qui implique un seul hommage dans l'intérêt du seigneur qui veut recevoir un service incompatible avec la division successorale (M. Garaud, Les châtelains du Poitou et l'avènement du régime féodal, Poitiers, 1967, p. 74 ; P. Ourlac. J. de Malafosse, Histoire du droit privé, Paris, $1968, \mathrm{t}$. III, p. 404-405). Mais ces facteurs d'explication restent bien limités. L'aînesse n'est que la concrétisation dans le droit de l'époque d'une réalité sociale qu'impose la militarisation des groupes privilégiés et la genèse des structures lignagères. 
et sur les terres du pays de l'étang de Berre. De son côté, Raimon Sacristain obtient un certain nombre de biens périphériques, situés autour de Sénas et au sud de la Camargue. Ici, le système de la melioratio, qui vise à avantager l'un des enfants dans les successions, apparaît de façon évidente, mais ce partage, qui se trouve à l'origine de la constitution des deux principales branches des Porcelet, n'est pas en réalité si net qu'un premier aperçu pourrait le laisser entendre. En fait, les Sacristain conservent encore des droits et des terres éparpillés à l'intérieur du domaine de la branche des Porcelet au sens propre, ce qui explique la solidarité des uns envers les autres. Les deux héritages restent souvent inextricablement mêlés ; les solidarités du cousinage ne souffrent certainement pas de cette division du patrimoine.

On a longtemps insisté sur la raison d'être économique du lignage. Certes, la fixation autour d'un patrimoine détenu en indivision joue un rôle essentiel dans sa genèse. Mais il n'en détermine pas tous les mécanismes. Le facteur militaire rend aussi bien compte des comportements démographiques de chacune des maisons nobiliaires. Au cours d'une période trouble, née de la mutation dite féodale, dans un milieu aristocratique qui parle, avant tout, le langage des armes, chaque lignage vise à augmenter le chiffre des chevaliers issus de ses rangs. Il ne saurait donc décourager la natalité ni la nuptialité des membres qui le composent. Des branches collatérales ou sous-lignages d'une puissance économique moindre que leur souche entrent alors dans le réseau de clientèles de chaque maison nobiliaire.

\section{Mentalités religieuses du lignage}

Les chartes de la période reflètent l'importance acquise par la parenté dans la vie de l'individu. Tous leurs éléments - depuis le préambule jusqu'à la liste des souscripteurs - font ressortir avec force cette solidarité entre les membres d'une même famille, attitude qui contraste nettement avec la liberté dont le couple conjugal jouissait à la fin $\mathrm{du} \mathrm{X}^{\mathrm{e}}$ siècle vis-à-vis de sa parentèle. Ces transformations vont de pair avec des changements dans le domaine des mentalités religieuses.

La lecture des préambules de l'acte, dans lesquels le scribe exprime une considération générale d'ordre juridique ou théologique, montre que les donateurs adoptent une nouvelle sensibilité à l'égard des lieux de culte. Par son geste, celui qui donne cherche à établir un lien artificiel de parenté avec la communauté religieuse qui se voit gratifiée de sa largesse. De nouvelles conceptions ecclésiologiques servent particulièrement son propos. Alors qu'une éthique du mariage et de la famille, plus en accord avec l'enseignement des Evangiles, est véhiculée par la réforme grégorienne 26 , les

26. Certains préambules mettent, par exemple, l'accent sur l'aspect contractuel du mariage et sur l'importance de cerlains rites pour le contracter: Ante tempus legi istius donationis etiam sine gestorum testificatione valebant; nunc vero, post hanc legem, nec nuptiali nec qualibet inter quascumque donatio valere potest si gestibus non fuisset alligata, CSV n 176 (18 II 1035). 
théologiens utilisent les images que leur fournit la réflexion des canonistes sur la parenté. L'Eglise apparaît en effet très souvent, dans ces préambules, en tant qu'épouse du Christ 27 , selon la comparaison paulinienne. Ce discours s'applique aussi pour chaque église particulière, dont le mariage est patronné par un laïc28. Chaque donneur entend apporter une dot à l'Eglise au sens large, tandis qu'il aide une nouvelle fondation ${ }^{29}$. Il entre ainsi de plein droit dans la fraternité d'une communauté religieuse et profite des biens spirituels qui en découlent, de même que n'importe quel individu reçoit alors la protection de son lignage 30 . Pour sceller cette adoption, le cloître se dessaisit souvent d'un bien symbolique en faveur du généreux donateur, car cette civilisation ne conçoit guère les relations, qu'elles soient humaines ou divines, que dans les termes du don et du contre-don 31 .

Après les préambules, les dispositifs des chartes nous plongent dans la réalité plus matérielle des noms et des origines des donneurs ainsi que de la nature et de la situation des biens cédés. Ils montrent que rares sont les donateurs qui agissent seuls. Chaque individu est entouré par ses parents, aussi éloignés soient-ils. Il serait inutile de revenir à nouveau sur les résultats de l'application d'une méthode statistique au cartulaire de SaintVictor ${ }^{32}$. A en croire les textes de ce fonds, les fortunes aristocratiques appartiennent au lignage tout entier, à cette réalité désignée par le nom de fratresca ${ }^{33}$. Il en va de même dans les actes du chapitre d'Arles relatifs aux zones rurales. La belle série de restitutions des dîmes et des églises situées au bord de l'étang de Berre présente vers 1080 un bon nombre de consanguins en train d'abandonner des terres qu'ils possèdent en commun ${ }^{34}$.

27. Jhesus ipse, virginis filius, in matemis visceribus humanam sumens naturam, sanctam sibi sociavit Ecclesiam, quam fidei anulo subarrans sicut propriam veste nubtiali vestivit sponsam, eamque eo dilexit quatenus pro ejus amore mortem subire non recusaverit, CSV $\mathrm{n}^{\circ} 325$ (15 XI 1019).

28. Sur ce patronage, voir J. HeERs, op. cit., p. 255.

29. Secundum ecclesiasticum morem, ut Christi sponse, concedimus in dotem..., CSV $n^{\circ} 268$ (1033) ; eam rogavi consecrari et ipsi in dotem et in sponsalicium medietatem de Cescuesta, quam tenebamus, donavi, CSV n 33 (1038-1048). Voir en outre CSV n 44 (1030), 112 (1056), 214 (28 I 1018), 293 (15 XII 1038), 412 (c. 1050).

30. Ego vero frater Petrus et monachi Sancti Victoris donamus tibi Fulcone nostram fratemitatem et societatem deprecantes Deum omnipotentem ut det tibi partem omnium bonorum fratrum nostrorum, CSV n ${ }^{\circ} 62$ (1053).

31. Uc cède un manse à Saint-Victor, partim pro ipso caballo et ipsa mula, partim vero pro remedio anime mee et pro remedio animarum patris mei et matris mee et parentum meorum, CSV $\mathrm{n}^{\circ}$ 80 (1048).

32. Cf. supra. Voici des exemples de quelques-uns de ces dispositifs : Ego Amalricus et uxor mea Rainvis et filii nostri et frater meus Guillelmus et nepotes mei Pontius Borellus et Pontius Abonellus et Boso et Beatrix mea neptis, CSV $n^{\circ} 51$ (1051); cum consilio frat rum et nepotum meorum, CSV n' 622 (18 VII ou 12 XII 1056); nos Audibertus et uxor mea Ermengarda filiusque domnus Amelius, miles, et frater meus Rostagnus necnon et nepotes mei Dodo, Upertus, Abellonius, Poncius, Qualo, Rostagnus, Arbaudus, P. AMArgIer, Chartes..., n ${ }^{\circ} 40$ (1052); Nos omnes pariter coheredes, CSV n 267 (15 X 1059).

33. De supradicto castro et de omnibus appendicis ejus que pertinent ad meam, ut ita dixerim, fratriscam, dono Sancto Victori, CSV n ${ }^{\circ} 57$ (1035).

34. Et ideo nos, scilicet Bermundus de Velaus et frater meus Petrus atque consobrini mei, scilicet Ugo et Gaufredus et uxores nostre et filii filieque nostre, J.-H. AlBANES, U. CHEVALIER, Gallia Christiana Novissima, Valence, 1901, t. III, n 441 (1082). 
Nul ne saurait détacher une partie du patrimoine familial sans le conser.. ment de ses proches.

Ces actes exposent, ensuite, les raisons pour lesquelles le donateur se dessaisit de l'un de ses biens. Le motif en est toujours d'ordre surnaturel car, en faisant l'aumône, l'on cherche avant tout le salut de son âme. Toutefois, les clauses pro anima écrites sur les parchemins sont rarement égocentriques. Le donneur prend habituellement soin d'y ajouter des demandes pour d'autres membres de son entourage. Ainsi, le nombre de donations qui ont été faites au XIe siècle en faveur de Saint-Victor pour obtenir la vie éter. nelle à titre purement personnel est négligeable (tableau 3). Elles représentent moins du cinquième des actes de son cartulaire. En revanche, le cercle restreint de la famille étroite - parents et enfants - occupe une place bien plus importante dans les demandes de suffrages (55 mentions sur 137). Mais, à partir des années 1020 , les aumônes faites pour le salut de toute la parentèle l'emportent systématiquement sur celles qui concernent le seul groupe des intimes : bel exemple encore du triomphe des structures lignagères et de leur influence sur les sensibilités religieuses ! Cette mutation intervient plus tôt en Provence que dans le Biterrois, où l'individualisme l'emporte pour plus de la moitié jusqu'en 1050. A partir de cette date, le donateur n'agit exclusivement pour son âme que dans le tiers des cas; il adjoint, pour un autre tiers, une mention de l'âme de ses père et mère et, pour un dernier tiers, de celle de tous ses parents ${ }^{35}$. Le modèle du Latium paraît bien divergent : les donations pieuses faites pour obtenir des suffrages en faveur de l'âme d'un parent extérieur à la famille conjugale sont rarissimes ${ }^{36}$. Ces constatations corroborent la place qu'occupe le cercle des parents les plus intimes dans les dernières pensées de l'homme de cette époque. L'étude des actes de la pratique révèle, ainsi, une attitude bien différente à l'égard de la mort que celle que proposait Philippe Ariès, à la seule lumière des sources littéraires 37.

Laissées pour compte de la recherche positiviste, les formules comminatoires reflètent bien des aspects de la religion populaire, impossibles à cerner par la seule analyse des dispositifs des chartes ou des chroniques. Ainsi, les scribes monastiques, toujours prévoyants, prennent bien soin d'implorer le ciel pour qu'il envoie les pires châtiments au membre de la famille

35. M. Gramain-Derruau, Villages et communautés..., p. 211.

36. P. TOUBERT, op. cit., p. 710.

37. "Comparons les dernières pensées du chevalier médiéval à celles des soldats de nos grandes guerres contemporaines, qui appelaient toujours leurs mères avant de rendre l'âme. Roland, lui, garde au seuil de la mort, le souvenir des biens possédés, des terres conquises, regrettées comme des êtres vivants, de ses compagnons, des hommes de sa bande, et du seigneur qui l'a élevé et qu'il a servi. (...) Dans les romans de la Table Ronde, la femme et l'enfant ont plus de place, mais les parents sont toujours oubliés "(L'homme devant la mort, Paris, 1977, p. 22). Contra : Ego (...) Ermengarda (...) cedo vel dono pro anima genitorio meo Pontione et matre mea Bellidrude vel pro anima mea Ermengarda, indigna peccatrice et pro anima filio meo Flavio, CSV n ${ }^{\circ}$ 169 (6 II 1000); pro redemptione mee anime, uxorisque mee karissime ac filii mei Nivonis, CSV $n^{\circ} 186(1010)$. 

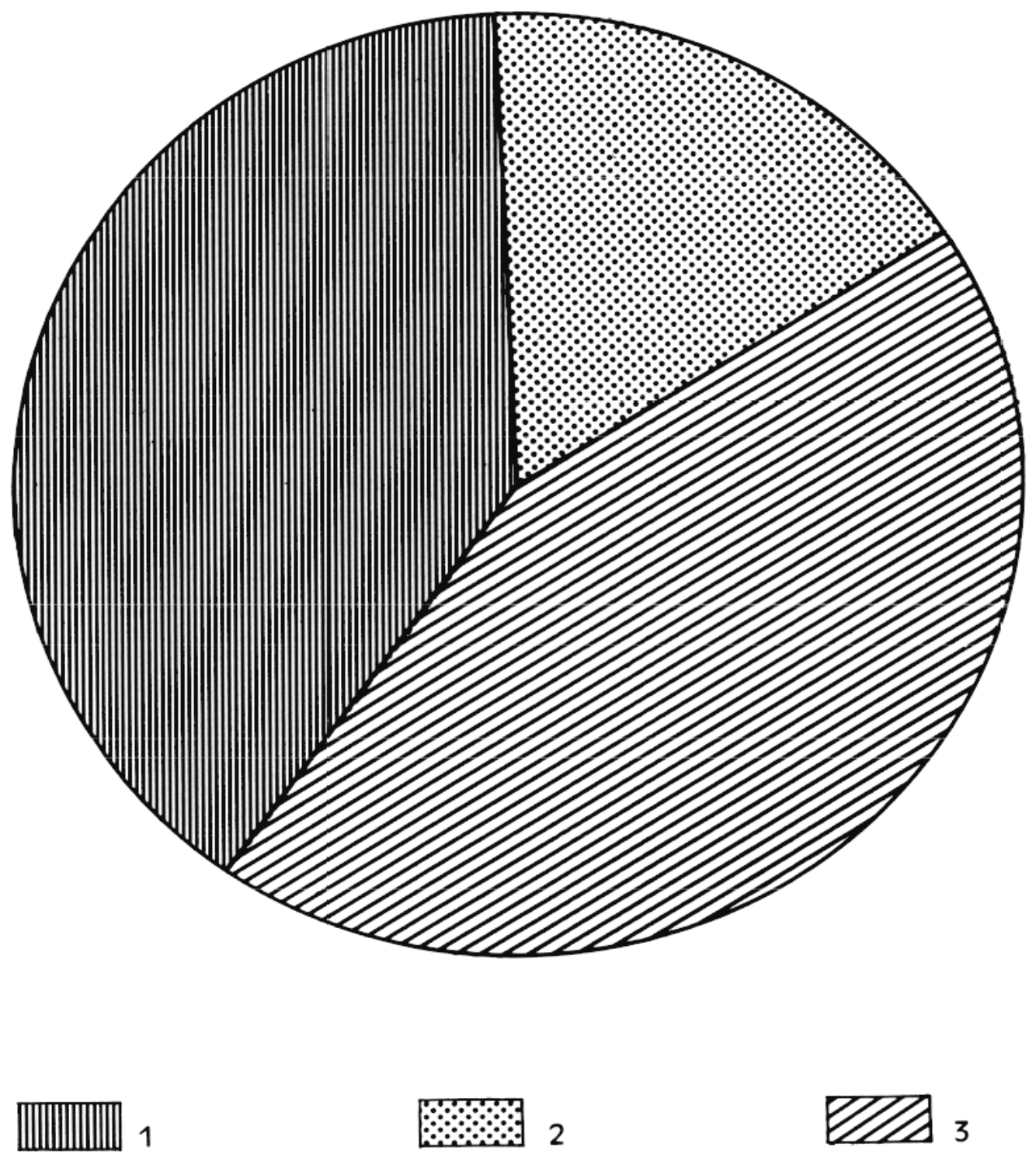

1 : Pour la famille étroite. 2 : Pour le donateur seul. 3 : Pour la famille élargie.

\begin{tabular}{|l|c|c|c|c|c|c|c|c|c|}
\cline { 2 - 10 } \multicolumn{1}{l|}{} & $\begin{array}{r}1000- \\
1009\end{array}$ & $\begin{array}{r}1010- \\
1019\end{array}$ & $\begin{array}{c}1020- \\
1029\end{array}$ & $\begin{array}{c}1030- \\
1039\end{array}$ & $\begin{array}{c}1040- \\
1059\end{array}$ & $\begin{array}{c}1059 \\
1060-\end{array}$ & $\begin{array}{c}1070- \\
1080\end{array}$ & Totaux \\
\hline $\begin{array}{l}\text { Pour le salut } \\
\text { de la propre ame }\end{array}$ & 3 & 3 & 3 & 6 & 3 & 3 & 1 & & 22 \\
\hline $\begin{array}{l}\text { Pour le salut } \\
\text { de ses proches } \\
\text { parents (parents } \\
\text { et enfants) }\end{array}$ & 5 & 5 & 6 & 7 & 9 & 13 & 5 & 5 & 55 \\
\hline $\begin{array}{l}\text { Pour le salut } \\
\text { de tous les parents }\end{array}$ & 1 & 6 & 7 & 12 & 9 & 14 & 9 & 2 & 60 \\
\hline Totaux & 9 & 14 & 16 & 25 & 21 & 30 & 15 & 7 & 137 \\
\hline
\end{tabular}

Tableau 3. - Les demandes de suffrages dans le Cartulaire de Saint-Victor. 
d'un donateur qui oserait enfreindre ses dispositions testamentaires ${ }^{38}$. Ils menacent les usurpateurs des peines de l'enfer, où ils rejoindront, entre autres, Datan, Abiron, Simon le mage et Judas Iscariote. Ces imprécations ne sont pas de style et les exemples de parents qui vont à l'encontre des dernières volontés de l'un de leurs consanguins sont si nombreux qu'à la fin du XIe siècle ils ont fait basculer une bonne partie des restitutions, qu'un élan premier de réforme avait rendues à l'Eglise, du côté des patrimoines aristocratiques ${ }^{39}$.

Une étude des chartes dans la perspective de l'histoire des mentalités ne manque donc pas d'intérêt. Elle permet, d'une part, de cerner le bagage intellectuel d'un scribe monastique du XI' siècle. Son discours apparaît alors fortement influencé par le mouvement grégorien. Ses textes défendent ainsi l'autonomie des lieux de culte vis-à-vis du pouvoir laïc, l'exemption épiscopale et la primauté de Rome. Sa réflexion sur la famille se fait l'écho de l'aspect contractuel de tout mariage, tandis que les canonistes affinent leurs conceptions sur cette institution. L'Eglise est présentée comme l'épouse du Christ seul et dégagée de toute emprise simoniaque. Ces mêmes documents aident, d'autre part, à percevoir quelques traits de la religiosité des aristocrates. Craignant le châtiment final, ceux-ci assurent leur salut en s'incorporant à une fraternité monastique ou en dotant une église. Bien que solidaires de leurs proches dans leurs prières, ils doivent faire face à l'opposition de ces mêmes parents qui cherchent à récupérer telle ou telle donation. Le modèle lignager est incompatible avec les aspirations d'un clergé réformé.

Les sources provençales du $\mathrm{XI}^{\mathrm{e}}$ siècle confirment l'ampleur du mouvement de cohésion lignagère au sein de l'aristocratie. Autour de 1020, tandis que le comté s'enlise dans une période d' " anarchie féodale », le vaste cousinage qui unissait des consanguins sur deux ou trois générations et sur un plan d'égalité se transforme. Cette structure horizontale est remplacée par un système vertical, où le patrimoine se transmet en bloc de père en fils. La primogéniture s'impose. Chaque lignée de guerriers se singularise. Elle adopte des comportements démographiques aptes à préserver le nombre de ses effectifs. Des taux de natalité et nuptialité élevés élargissent la base des fidèles insérés dans les réseaux de clientèle d'un lignage. Une telle mutation transparaît également dans les mentalités nobiliaires. Soucieux de son salut et de celui de toute sa maison, l'aristocrate cherche à s'intégrer dans un lignage ecclésiastique. Ainsi, les solidarités familiales dépassent le profane pour rejoindre le sacré ; elles perdurent dans l'au-delà.

38. Si quis vero heredum nostrorum vel quilibet hominum hac donationem violare temptaverit non valeat..., P. AMARGIER, Chartes..., $\mathrm{n}^{\circ} 16$ (1045); Sine ulla interpellatione aliquorum nostrorum heredum, masculorum et feminarum, consaguineorum vel aliquorum hominum, $\mathrm{CSV} n^{\circ} 78(1048)$.

39. Sur les contestations de l'aristocratie provençale au sujet des donations des membres de leurs familles, voir G. Demians d'Archimbaud, Les fouilles de Rougiers, Paris-Valbonne, 1980, p. 32-33, et S. Weinberger, Les conflits entre clercs et laïcs dans la Provence du XIe siècle, dans Annales du Midi, 1980, p. 269-280. 